\title{
Integration of Algorithm-Based Optimization into the Design Process of Industrial Buildings: A Case Study
}

\author{
Mirjam Konrad ${ }^{(凶)}$, Dana Saez ${ }^{(凶)}$, and Martin Trautz \\ Chair of Structures and Structural Design, RWTH Aachen University, Schinkelstr. 1, \\ 52062 Aachen, Germany \\ mirjam.konrad@rwth-aachen.de, saez@trako.arch.rwth-aachen.de
}

\begin{abstract}
Algorithm-based optimization is widely applied in many fields like industrial production, resulting in state-of-the-art workflows in the production process optimization. This project takes the cultural lag of conventional industrial architecture design as a motivation to investigate the implementation of algorithmbased optimization into traditional design processes. We argue that an enhanced way of architectural decision-making is possible. Current approaches use a translation of the whole design problem into a single, overly complicated optimization system. Contrary to that, this paper presents a novel workflow that defines precise design steps and applies optimizations only if suitable. Furthermore, this method can generate relevant results for factory planning design problems with contradicting factors, making it a promising approach for the complex challenges of i.e. resource-efficient building.
\end{abstract}

Keywords: Algorithm-based optimization - Evolutionary Multi-objective Optimization · Industrial architecture $\cdot$ Architectural design $\cdot$ Design methodology

\section{Introduction on Form Finding}

Designing from the abstract to the concrete implies a series of different transformation, evaluation, and decision-making processes [2]. In traditional design processes the background or intuition of the designer serves as a basis for the decision-making of the iterative actions that give form to the object of design. Although the design process is generally non-linear, it renders strict, systematic methods that hamper an integral part of the process: experimentation [3]. Nowadays, facing the information age, decisionmaking and all its facets are increasingly challenging to obtain. In the past, interdisciplinary approaches to design strategies have proven to be successful. The interactions between computer science, mathematics, and architecture have led to the invention of CAD software, for example, from which Building Information Modeling (BIM) or parametric applications have evolved. However, the spread of new technologies, such as form-finding tools, is very slow, and their utilization in the architectural design process 
is scarce [14]. New digital methods are no longer merely a digitalization of the conventional analog planning process. They could also be implemented into design processes to enhance the decision-making workflow when working with complex programs as industrial facilities.

\subsection{Form Finding in Industrial Architecture}

Industrial production is known for its advance in process optimization, economic efficiency, and hierarchical structures. Hence, factory planning requires a different design approach than conventional projects. A specific, clearly defined program, its possible expansions, and the detailed data of existing productions should be considered. The goal of economic efficiency should not only be achieved by optimizing production processes but also by planning the building structure and equipment [10]. Typically, the production design and the building design occur separately and interact too late so that the building results into a polygonal envelope of the simplified space program [9]. Concerning architecture, the continuous optimization of production and the extensive collection of data regarding utilization and profitability, is in severe contrast to the planning and design of the factory building [9]. Production technology is undergoing increasingly rapid change. The advent of automation and partial automation of work steps has shown that the demands placed on production spaces can change several times during the building's service life [10]. Current box-in-box principles are often used as a reaction but require factory constructions with the broadest possible, column-free enclosed space. That is why space shortage and, above all, sustainability and resource economy represent a considerable challenge for contemporary industrial architecture.

It is worth considering what the digitalization and interdisciplinary aspects of the industry have to offer and question the current architectural planning process. This research project pursues an alternative approach, investigating how to integrate evolutionary single- or multi-objective optimizations into the design processes of industrial architecture. The aim is to solve specific design decisions, i.e. production process layout or accessibility of factory spaces, based on the provided data.

\subsection{Architecture Optimized by Algorithms}

Algorithm-based optimization is one of the technologies used to improve architectural designs. An algorithm could be defined as a set of rules consisting of distinct, finite steps to solve a problem [13]. Supposing one considers the design task a problem of partially contradictory influencing factors, which are in equilibrium in an optimal state, an algorithm can be used to find a potential solution. Optimization principles are implemented by parameters and their relations defined by design factors and constrains. An algorithmoptimized architecture can be archived by solving the problem concerning one or more defined goals by optimizing the parameters. Thus, the design derives from its restrictions and demands on the space. This bottom-up process allows architects to influence the design even without knowing the final shape [1]. By generating solutions that are neither known to the designers nor imaginable for them, a new way of decision-making is created [15] (Fig. 1). 

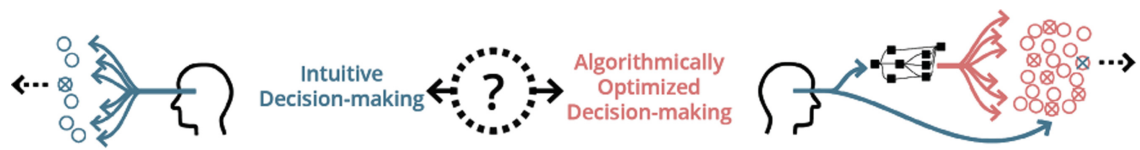

Fig. 1. Differences in decision-making

\section{Methodology}

In industrial design projects, data is usually available regarding the production process. Within this project's scope that deals with a new type of serial production of natural fiber composite dinghy hulls, this data was established by the author. It consists of an organizational chart showing the various relationships between the production steps and room data sheets. The latter lists the requirements for the production steps as well as room specifications regarding minimum dimensions, operational safety, and work environment.

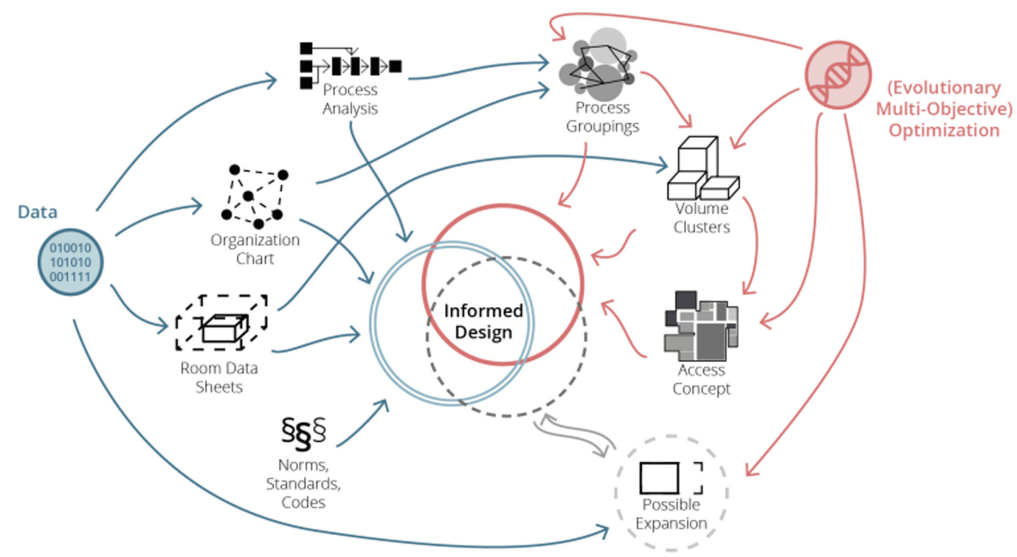

Fig. 2. Diagram of the overall methodology of informed design

In order to implement algorithm-based optimizations, a simple translation of design requirements into a single, overall optimization system was not a viable approach. Therefore, the design steps were formulated and dissected to investigate which could benefit from the optimization application.

Figure 2 shows how the data and optimization influenced the so-called informed design process. Whether digitally enhanced or not, each design decision formed the basis of the next and most directly made use of the data provided. In this way, the method was sensibly developed from the global to the detail. The aim was to find a method where human design skills and digital tools can work together. The key of the process was to implement specific tools readily available in a transparent way so it can be adapted to other factory design projects. 


\subsection{Overview of the Design Steps}

All optimizations were implemented with the CAD software Rhinoceros $3 D$ in the visual programming environment Grasshopper. They were partly based on existing plug-ins or, i.e. the volume cluster optimization, explicitly developed for this research (Fig. 3).

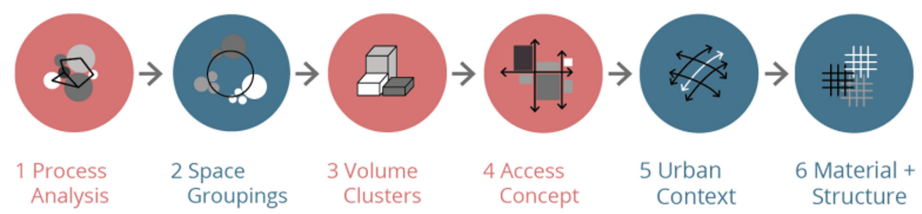

Fig. 3. Design steps. Digitally optimized decision-making steps in red, conventional design steps in blue

Process Analysis. The process structure was developed, employing the Grasshopper Plug-In Syntactic by Pirouz Nourian, which offered a toolset that generated a function graph via points and lines using a force-based graph drawing algorithm [12]. Production steps and their significance based on the frequency of use and their connection were input into the optimization. The graph was then displayed as a bubble diagram. A catalog summarized the resulting diagram-like solutions to develop a model of the process structure.

Space Groupings. Based on the process organization, the production steps were manually grouped into areas.

Volume Cluster. This step will be further explored in Sect. 2.2.

Access Concept. Since an efficient production relies on an adequate access concept, the previously generated abstract volume clusters' arrangement was optimized. The basis for this optimization was the Grasshopper Plug-In Magnetize, a project by Egor Gavrilov, which creates an optimal corridor system that links rectangular, two-dimensional spaces based on previously specified, necessary relations between said spaces [4]. The footprints of the abstracted volume clusters were arranged by the optimization and provided with optimal access. The generated floor plans were analyzed to form rules, which were then applied to the design.

Urban Context. Intuitive but informed decision-making strategies were used to situate the building in the urban context. For this purpose, all findings from the design steps and the SWOT analysis were consulted. By combining these principles, an overall cluster was produced, which can function as the starting point of the formulation.

Material and Structure. The final step was the design of the construction and the choice of materials. Following the project, which focuses on resource-saving boat building, sustainable materials were prioritized. Often, however, the function of the room determines the materials used. 


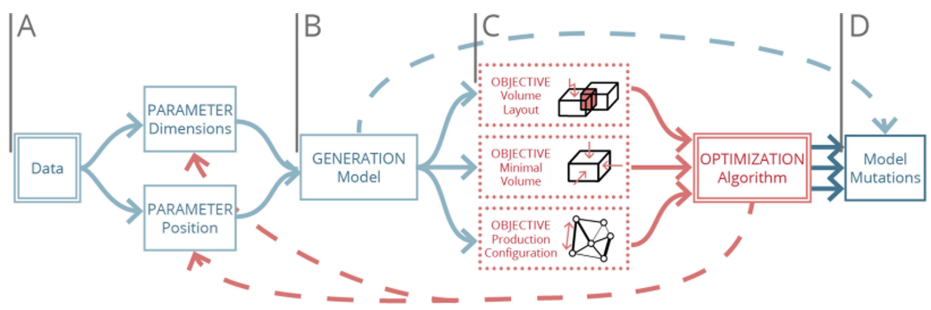

Fig. 4. Simplified functionality of the volume clustering optimization

\subsection{Volume Clustering Optimization}

Using the Evolutionary Multi-objective Optimization Octopus, [16] not all optimization factors had to be calculated into a single optimal state, but solutions were generated according to several criteria. Thus, it remained comprehensible why options were classified as better, which facilitated the decision process. The data was processed to determine the numerical target value over several steps using the following Grasshopper script [5-8, 11] (Figs. 4 and 5).

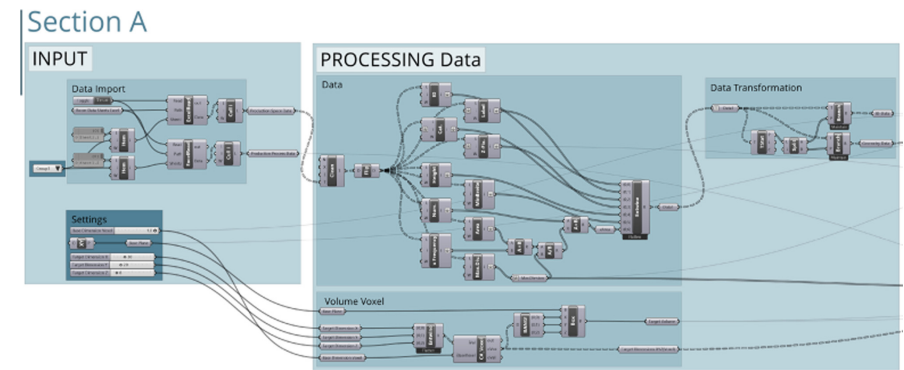

Fig. 5. Section A. Volume clustering optimization script in Grasshopper/Rhinoceros $3 D$

The aim was to optimize the clustering of selected production steps, represented by abstract boxes, into a target box. Consequently, they were simplified by using voxels as a base unit. Section A of the script was responsible for selecting the main settings, such as voxel size and global target dimensions. It also handled the data import of the production spaces and the processing, mainly splitting the data into identity-related and geometry-related data (Fig. 6).

In Section B the optimizable parameters were generated and provided the basis for the first modelling. The boxes' form was defined by creating dependencies between the imported, fixed values room height, minimum depth and volume of each production space, and the variable width $x$ while factoring in the maximal division value of the spaces. Then the parameters $x, y$, and $z$ for the positioning of the space were defined. Finally, gene pools were set for these two parameters, determining the location and the width. 


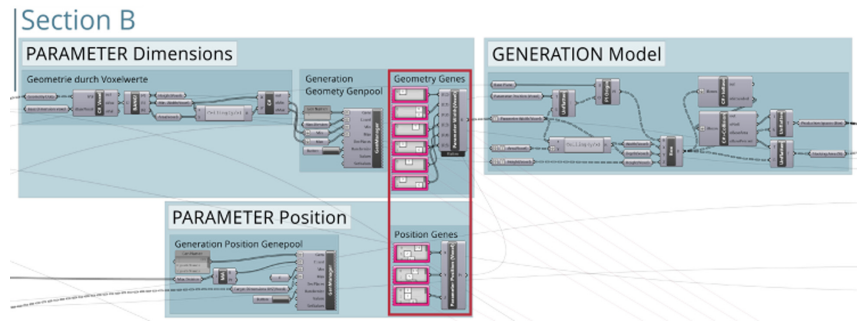

Fig. 6. Section B. Volume clustering optimization script in Grasshopper/Rhinoceros $3 D$

The model of the production spaces was generated using the genes. This was scripted using an iterative method of first sorting the boxes decreasingly by volume and then stacking them on top (Fig. 7).

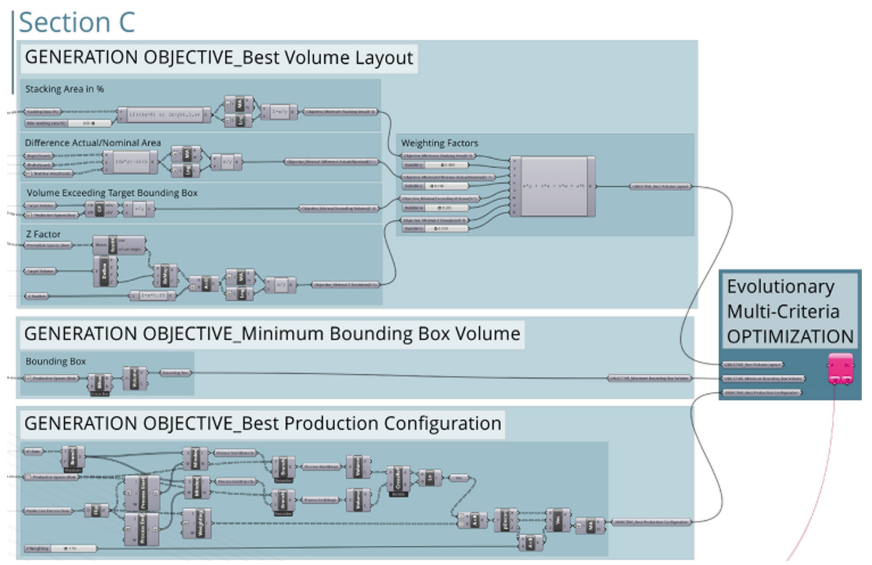

Fig. 7. Section C. Volume clustering optimization script in Grasshopper/Rhinoceros $3 D$

From this model, the three target values were then determined in Section C, towards which the optimization was aimed. The best volume layout objective pursues the most realistic stacking and arrangement of the voxel volumes. It is obtained by adding the normalized and weighted values of several factors, such as the minimum stacking area, the volume exceeding the target bounding box, the preferred proximity of the space to the base plane, and the actual and nominal footprint difference.

The objective minimum bounding box volume numerically represents the size of the bounding box of the generated volume. The objective best production configuration represents the sum of the distances of the connections between spaces, weighted by their priority. The evolutionary optimization Octopus was executed to manipulate the given gene pools to minimize the target values. After generating the first population of solutions, the following child generations were subsequently derived through selection, crossing, and mutation. 


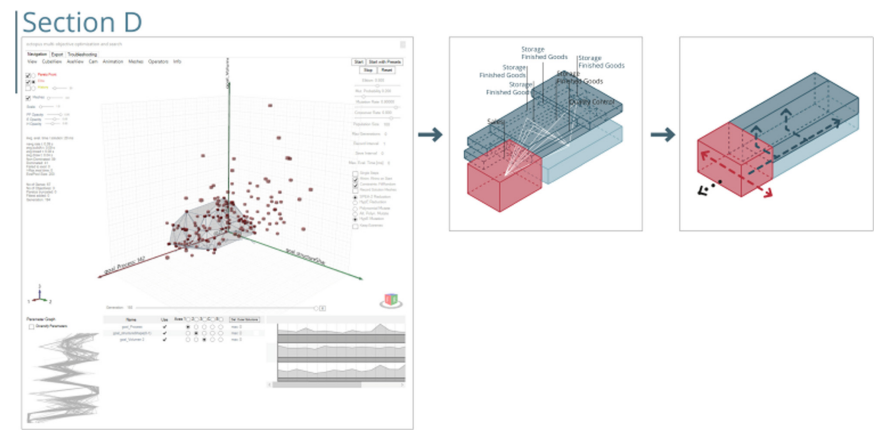

Fig. 8. Section D. Volume clustering optimization in Octopus/Rhinoceros $3 D$

In Section D (Fig. 8), a pool of solutions was created by repeating the optimization for a sufficient number of generations. The best solutions were analyzed by using the pareto mesh of the three objectives. The individual final clusters and their iterations were examined for their functional principles of spatial arrangement, e.g., vertical stacking or prefixed volumes. Finally, these principles or arrangement schemes were incorporated into the design.

\section{Results}

This research project integrates algorithm-based optimizations into the design process of an exemplary industrial architectural project to improve the coherence of the production and the factory building. Using the developed design method, an arrangement of symbiotic production rooms in the final architectural design was achieved, providing proof of concept.

Figure 9 (left) shows the design decisions derived from either data processed by optimizations or conventional design techniques. The diagrams on the right display retrospectively the influences of these decisions on the final structure. The results, as shown in Fig. 9, indicate that the production of the factory and its form are connected within all the design steps.

\section{Discussion and Future Development}

The results indicate the benefits of defining precise design steps and applying optimizations if suitable. Compared to the conventional process, the proposed system is able to utilize an extensive amount of data provided to improve the outcome. It is possible to generate relevant results, even for design problems with contradicting factors.

The effectiveness of the suggested method stems from its adaptability. Contrary to many concepts in parametric design, this approach can handle complicated and straightforward problems. By breaking the design task down into steps, the problem can be simplified, and the solutions can individually be implemented into the design process.

The presented design method developed in this case study should be understood as a guideline, as it was not intended to create a design generation tool. A proper application 

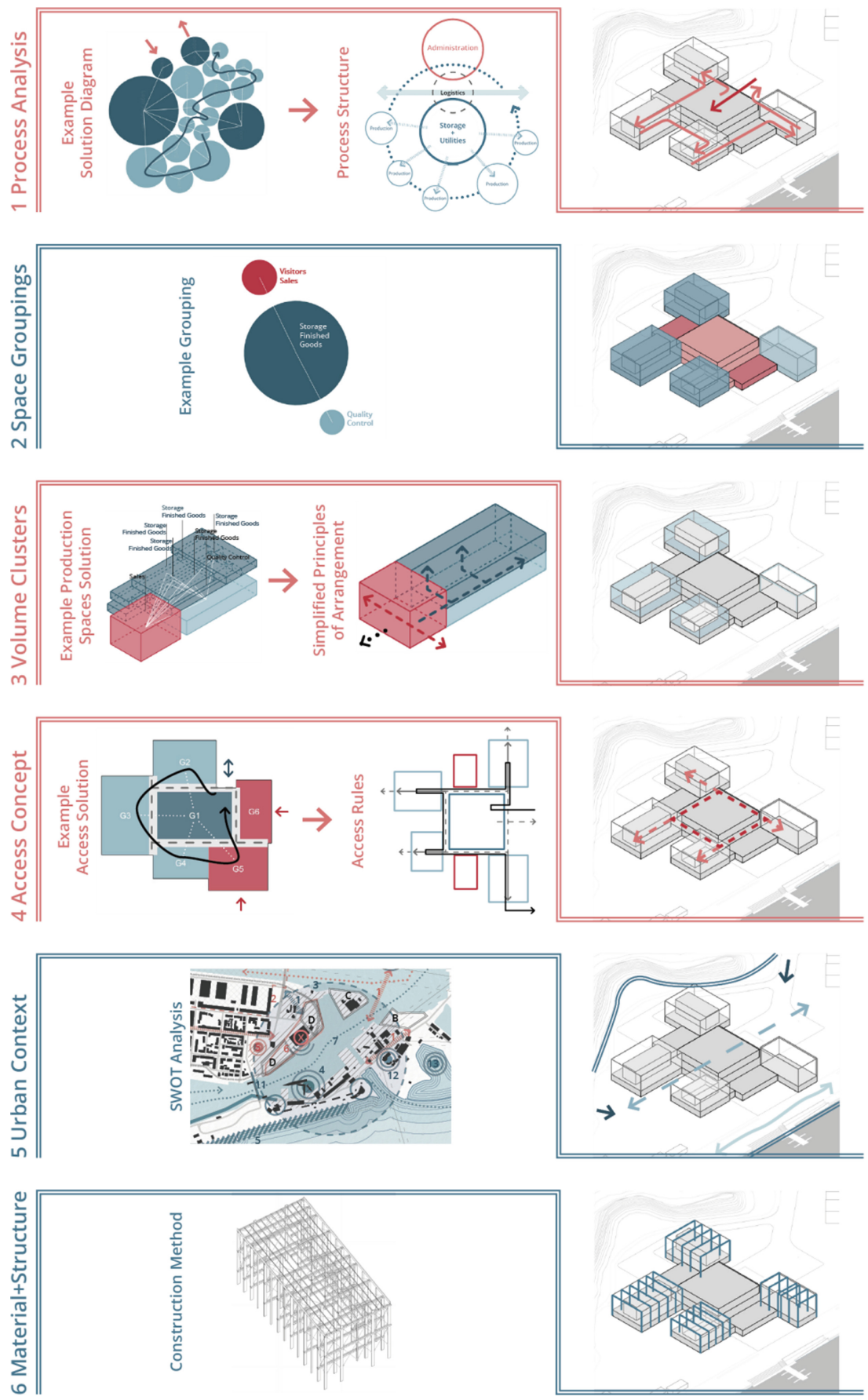

Fig. 9. Results of the design steps and their representation in the final design. Digitally optimized decision-making steps in red, conventional design steps in blue 
of optimizations can only be recommended if the limitations and operating methods are known and not hidden as a black box application. The abstract logic inherent in technologies such as optimizations can promote and improve conventional design, as long as their use is transparent and plausible.

Another interesting benefit is the possibility of using the design structures after completion of the planning or construction. An evaluation of built designs through their designing factors could provide crucial information of performance and degree of capacity utilization. This could become a digital twin of the functioning, making projections for expansions or restructuring more precisely.

\section{Conclusion}

As parametric design has proven to be valuable and effective after years of practical use, optimization should be applied more often in industrial architecture. Optimized and conventional design steps should not be seen as contradictory or in competition with each other. Instead, their differences offer opportunities to tackle problems in different ways. The outcome of this project shows that they work particularly well in combination.

This new design process could be an essential element for responsible and sustainable factory planning. In today's age of information, optimizations offer technology that, using available data, could solve the complex problems of resource-efficient building and should therefore be investigated further.

\section{References}

1. Azambuja Varela, P.: Genetic algorithms in architecture. In: Sousa, J.P., Xavier, J.P., ECAADE (Association), Universidade do Porto, Faculdade de Arquitectura (eds.) Future Traditions: Rethinking Traditions and Envisioning the Future in Architecture Through the Use of Digital Technologies. Proceedings of the 1st ECAADe Regional International Workshop. FAUP Publicações, Porto (2013)

2. Bielefeld, B., Khouli, S.: Entwurfsidee, Rev. Birkhäuser, Basel (2011)

3. Dursun, P.: Space syntax in architectural design. In: Proceedings of the 6th International Space Syntax Symposium (2007)

4. Gavrilov, E.: Magnetizing floor plan generator (2019). https://www.food4rhino.com/app/mag netizing-floor-plan-generator. Accessed 5 Mar 2021

5. Heimig, T.: GeneManager (2020)

6. Heimig, T.: GH_Pack (2020)

7. Heumann, A.: Human (2019). https://www.food4rhino.com/app/human. Accessed 5 Mar 2021

8. Heumann, A.: MetaHopper (2018). https://www.food4rhino.com/app/metahopper. Accessed 5 Mar 2021

9. Holzabsatzfonds (ed.): Industrie- und Gewerbebau in Holz (2008)

10. Laviola, C., Rustom, S.: Planungsleitfaden Zukunft Industriebau. Teil E: Strukturen zukunftsfähiger Industriebauten. Fraunhofer IRB Verlag, Stuttgart (2011)

11. Miller, N.: LunchBox (2016). https://www.food4rhino.com/app/lunchbox. Accessed 5 Mar 2021

12. Nourian, P.: SYNTACTIC (2018). https://www.food4rhino.com/app/syntactic 
13. Rogers, H.: Theory of Recursive Functions and Effective Computability, 1st MIT Press pbk. edn. MIT Press, Cambridge (1987)

14. Schulitz, H.C.: Form Follows Performance: Schulitz Architekten: Works 1995-2010= Leistungsform: Schulitz Architekten: Arbeiten 1995-2010, 1st edn. Birkhäuser, Basel, Boston (2010)

15. Terzidis, K.: Algorithmic Architecture, 1st edn. Architectural Press, Amsterdam, Boston (2006)

16. Vierlinger, R.: Octopus (2018). https://www.food4rhino.com/app/octopus. Accessed 15 Apr 2020

Open Access This chapter is licensed under the terms of the Creative Commons Attribution 4.0 International License (http://creativecommons.org/licenses/by/4.0/), which permits use, sharing, adaptation, distribution and reproduction in any medium or format, as long as you give appropriate credit to the original author(s) and the source, provide a link to the Creative Commons license and indicate if changes were made.

The images or other third party material in this chapter are included in the chapter's Creative Commons license, unless indicated otherwise in a credit line to the material. If material is not included in the chapter's Creative Commons license and your intended use is not permitted by statutory regulation or exceeds the permitted use, you will need to obtain permission directly from the copyright holder.

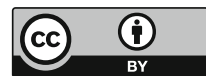

